Military Technical College, Kobry El-Kobbah, Cairo, Egypt

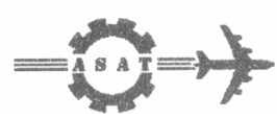

$9^{\text {th }}$ International Conference

On Aerospace Sciences \& Aviation Technology

\title{
RESTRICTIVE PADE' APPROXIMATION FOR SOLVING FIRST- ORDER HYPERBOLIC SYSTEMS IN TWO SPACE DIMENSIONS
}

\author{
By
}

Hassan N. A. Ismail and Adel Y. H. Elmekkawy"

\section{Abstract}

In this paper, we shall develop a new approach to an implicit method for solving the first-order hyperbolic systems in two space dimensions. The suggested method gives highly accurate result. The stability condition and the advantages of the considered method compared with the classical methods as Crank-Nicolson method are discussed.

\section{KEY-WORDS}

Pade`approximation, Restrictive Pade`approximation, finite difference and hyperbolic partial differential equations.

-Benha Higher Institute of Technology, Benha, Egypt

"Dept of Math. Faculty of Education, Ain Shams University 


\section{Introduction}

Consider the first-order hyperbolic systems of equations

$$
\frac{\partial u}{\partial t}=A \frac{\partial \underline{u}}{\partial x}+B \frac{\partial \underline{u}}{\partial y}
$$

where $A$ and $B$ are $n \times n$ real symmetric constant matrices, and $\underline{u}(x, y, t)$ is an $n-$ component column vector.

In this paper we define an implicit method for solving the first-order hyperbolic systems in two space dimensions, it produces very high accuracy compared with the other classical methods, i.e. the numerical solution produced by the considered method is almost identical to the exact solution. In previous work, we use the restrictive Pade' approximation as done in [2] to approximate the exponential matrix. Also, we use the restrictive Pade approximation, in [5] to approximate solution of first order Hyperbolic partial differentia0l Equations, in [6] to approximate solution of first order hyperbolic systems in one space dimension, in [7] to approximate solution of first order hyperbolic systems in two space dimensions.

\subsection{Restrictive Pade' Expansion (RPA)}

The restrictive Pade' approximation of the function $f(x)$ can be written in the form as done in [3]

$$
R P A[M+\alpha / N]_{f(x)}(x)=\frac{\sum_{i=0}^{M} a_{i} x^{i}+\sum_{i=1}^{\alpha} \varepsilon_{i} x^{M+i}}{1+\sum_{i=1}^{N} b_{i} x^{i}}
$$

where $\alpha=0(1) N, N$ is the degree of the numerator.

$$
f(x)-R P A[M+\alpha / N]_{f(x)}(x)=o\left(x^{M+N+1}\right) .
$$

Let $\mathrm{f}(\mathrm{x})$ have a Maclaurin series, as $\quad f(x)=\sum_{i=0}^{\infty} c_{i} x^{i}$

Then,

$$
\left(\sum_{i=0}^{\infty} c_{i} x^{i}\right)\left(1+\sum_{i=1}^{N} b_{i} x^{i}\right)-\left(\sum_{i=0}^{M} a_{i} x^{i}\right)-\left(\sum_{i=0}^{a} \varepsilon_{i} x^{i+M}\right)=o\left(x^{M+N+1}\right) .
$$

The vanishing of the first $(M+N+1)$ powers of $x$ on the left hand side of $(4)$ implies a system of $(M+N+1)$ equations, and hence we can determine the coefficient $c_{i}, a_{i}$ and $b_{i}$ as a function of $\varepsilon_{i}, i=1(1) \alpha$, where the parameters $\varepsilon_{i}$ are to be determined, such that

$$
f\left(x_{i}\right)=R P A[M+\alpha / N]_{f(x)}\left(x_{1}\right), \quad i=1(1) \alpha
$$

It means that the considered approximation is exact at $(\alpha+1)$ points. 


\subsection{Numerical Example of Restrictive Pade`Approximation}

The function $f(x)=\left(\frac{1+0.5 x+0.25 x^{2}}{1+5 x}\right)^{0.5}$,

its Pade' approximation and restrictive Pade' approximation takes the forms: $P A[2 / 1]_{f(x)}(x)=\frac{1+1.9311 x-0.563724 x^{2}}{1+4.1811 x}$,

$R P A[2 / 1]_{f(x)}(x)=\frac{1+1.73134 x-0.114257 x^{2}}{1+3.98134 x} \quad$ where $\alpha=1$ and $x_{\alpha}=0.6$

\section{Restrictive Pade' Approximation for the First-Order Hyperbolic Systems} in Two Space Dimensions

Consider the first-order hyperbolic systems of n-equations (1). The exact solution of its grid representation of equations(1) is:

$$
\begin{aligned}
\underline{u}_{i, j}^{m+1} & =\exp \left(k \frac{\partial}{\partial t}\right) \underline{u}_{i, j}^{m}=\exp \left(k\left(A \frac{\partial}{\partial x}+B \frac{\partial}{\partial y}\right)\right) \underline{u}_{i, j}^{m}, \\
\text { i.e. } \quad \underline{u}_{i, j}^{m+1} & =\exp \left(r\left(A D_{x}+B D_{y}\right)\right) \underline{u}_{i, j}^{m}
\end{aligned}
$$

where $D_{x} \underline{u}_{i, j}^{m} \cong\left(\underline{u}_{i+1, j}^{m}-\underline{u}_{i-1, j}^{m}\right)$ and $D_{y} \underline{u}_{i, j}^{m} \cong\left(\underline{u}_{i, j+1}^{m}-\underline{u}_{i, j-1}^{m}\right), \quad \Delta x=\Delta y=h, r=\frac{k}{2 h}$ The restrictive Pade' approximation [1/1] of the exponential matrix can take the form: $R P A[1 / 1]_{\exp \left(\left(r\left(A D_{x}+B D,\right)\right)\right.}(r)=\left(I+\left(\mathcal{E}-\frac{1}{2}\left(A D_{x}+B D_{y}\right)\right) r\right)^{-1}\left(I+\left(\mathcal{E}+\frac{1}{2}\left(A D_{x}+B D_{y}\right)\right) r\right)$

$$
\text { where } I \text { is the unit matrix of }(n \times n) \text { and } \varepsilon=\left[\begin{array}{llllll}
\varepsilon_{1 i} & & & & & 0 \\
& \varepsilon_{2 i} & & & \\
& & \varepsilon_{3 i} & & \\
& & & \ddots & \\
0 & & & & \varepsilon_{n i}
\end{array}\right] \text {, }
$$

we use equation (7) to approximate the exponential matrix in equation (6) as: $\underline{u}_{i, j}^{m+1}=\left(I+\left(\varepsilon-\frac{1}{2}\left(A D_{x}+B D_{y}\right)\right) r\right)^{-1}\left(I+\left(\varepsilon+\frac{1}{2}\left(A D_{x}+B D_{y}\right)\right) r\right) \underline{u}_{i, j}^{m}$.

In the case of, $A=\left[\begin{array}{ll}a_{1} & a_{2} \\ a_{2} & a_{3}\end{array}\right], \quad B=\left[\begin{array}{ll}b_{1} & b_{2} \\ b_{2} & b_{3}\end{array}\right]$, and $\underline{u}=(f, g)^{T}$

its solution consists of the two functions $f(x, y, t)$ and $g(x, y, t)$. They satisfy the initial and boundary conditions: 
$f(x, y, 0)=F(x, y)$ and $g(x, y, 0)=G(x, y), 0 \leq x, y \leq 1$

$f(0, y, t)=F_{0}(y, t), g(0, y, t)=G_{0}(y, t), f(1, y, t)=F_{1}(y, t)$, and $\left.g(1, y, t)=G_{1}(y, t), t \geq 0\right\}$

$f(x, 0, t)=F_{2}(x, t), g(x, 0, t)=G_{2}(x, t), f(x, 1, t)=F_{3}(x, t)$, and $g(x, 1, t)=G_{3}(x, t), \quad t \geq 0$

In this case, equations ( 8 ) are equivalent to the form:

$$
\left.\begin{array}{r}
\left(1+r \varepsilon_{i i}\right) f_{i, j, m+1}-\frac{r a_{1}}{2}\left(f_{i+1, j, m+1}-f_{i-1, j, m+1}\right)-\frac{r a_{2}}{2}\left(g_{i+1, j, m+1}-g_{i-1, j, m+1}\right) \\
-\frac{r b_{1}}{2}\left(f_{i, j+1, m+1}-f_{i, j-1, m+1}\right)-\frac{r b_{2}}{2}\left(g_{i, j+1, m+1}-g_{i, j-1, m+1}\right) \\
=\left(1+r \varepsilon_{1 i}\right) f_{i, j, m}+\frac{r a_{1}}{2}\left(f_{i+1, j, m}-f_{i-1, j, m}\right)+\frac{r a_{2}}{2}\left(g_{i+1, j, m}-g_{i-1, j, m}\right) \\
+\frac{r b_{1}}{2}\left(f_{i, j+1, m}-f_{i, j-1, m}\right)+\frac{r b_{2}}{2}\left(g_{i, j+1, m}-g_{i, j-1, m}\right)
\end{array}\right\}
$$

where $r=\frac{k}{2 h}, \quad i, j=1(1) n$ and $m$ non-negative integer.

We note that $\varepsilon_{1 \mathrm{i}}=\varepsilon_{2 i}=0$ gives Crank-Nicolson method. To determine the restrictive parameters $\varepsilon_{1 i}, \varepsilon_{21}$, we must have the exact solution at the first level, this enables the value of $f(x, y, t)$ and $g(x, y, t)$ at the grid points. Practically we found that $\varepsilon_{1 i}=\varepsilon_{21}$, hence we can say that $\varepsilon_{11}=\varepsilon_{11}=\varepsilon_{i 1}$.

\section{The Stability Analysis}

A Von Neumann stability analysis must considered the finite difference equations(10) and(11). This is accomplished by substituting the Fourier components of $\underline{u}_{i, j}^{m}$ as $\underline{u}_{i, j}^{m}=\underline{U}^{m} e^{I \beta h_{i}} e^{I \gamma h j}, \underline{U}=(F, G)^{T}$ where $I=\sqrt{-1}, \underline{U}^{m}$ is the amplitude at time level $\mathrm{m}$, and $\beta, \gamma$ are the wave numbers in the $\mathrm{x}, \mathrm{y}$ directions respectively. If a phase angles $\theta=\beta$ h and $\phi=y h$ are defined, then $\underline{u}_{i, j}^{n}=\underline{U}^{n} e^{i \theta_{i}^{i}} e^{I \phi^{j j}}$. A stability analysis of a hyperbolic systems in two space dimensions is very difficult, even $A$ and $B$ are constant, unless $A$ and $B$ commute, i.e. $a_{3}=a_{1}$ and $b_{3}=b_{1}$, which is the case in practice. The eigenvalue of the amplification matrix corresponding to the finite difference equations $(10)$ and(11) is:

$$
\lambda=\frac{\left(1+r \varepsilon_{i}\right) \pm I\left[\left(r a_{1} \sin \theta+r b_{1} \sin \phi\right) \pm\left(r a_{2} \sin \theta+r b_{2} \sin \phi\right)\right]}{\left(1+r \varepsilon_{i}\right) \pm I\left[\left(r a_{1} \sin \theta+r b_{1} \sin \phi\right) \pm\left(r a_{2} \sin \theta+r b_{2} \sin \phi\right)\right]}, I=\sqrt{-1} .
$$


i.e. $|\lambda|=1, \forall \varepsilon_{i}, r$ and $i=1(1) n$. Consequently the considered method is unconditionally stable.

\section{Local Truncation Error Upper Bound}

Using Taylor expansion, and after very long and computations we can obtained the local truncation error of the difference equation $(10)$ as the general form:

$$
\begin{aligned}
T_{i, j}^{(1)} & =\frac{1}{k}\left(1+\frac{\varepsilon k}{2 h}\right) \sum_{n=1}^{\infty}\left(\frac{k^{n}}{n !} \frac{\partial^{n} u}{\partial t^{n}}\right)_{(i, j)} \\
& -\frac{1}{h} \sum_{n=0}^{\infty}\left(\frac{h^{2 n+1}}{(2 n+1) !}\right)\left(b_{1} \frac{\partial^{2 n+1} f}{\partial y^{2 n+1}}+b_{2} \frac{\partial^{2 n+1} g}{\partial y^{2 n+1}}+a_{1} \frac{\partial^{2 n+1} f}{\partial x^{2 n+1}}+a_{2} \frac{\partial^{2 n+1} g}{\partial x^{2 n+1}}\right)_{(i, j)} \\
& -\frac{1}{2 h} \sum_{m=0}^{\infty}\left(\frac{h^{2 m+1}}{(2 m+1) !}\right) \sum_{n=1}^{\infty}\left(\frac{k^{n}}{n !}\right)\left(b_{1} \frac{\partial^{2 m+n+1} f}{\partial y^{2 m+1} \partial t^{n}}+b_{2} \frac{\partial^{2 m+n+1} g}{\partial y^{2 m+1} \partial t^{n}}+a_{1} \frac{\partial^{2 m+n+1} f}{\partial x^{2 m+1} \partial t^{n}}+a_{2} \frac{\partial^{2 m+n+1} g}{\partial x^{2 m+1} \partial t^{n}}\right)_{(i, j)} .
\end{aligned}
$$

Then if there exists a positive real numbers $M_{1}, M_{2}, M_{3}$ for all sufficiently large positive integer $\mathrm{n}$ such that

$$
\begin{aligned}
& \left|\frac{\partial^{n} u}{\partial t^{n}}\right|<M_{1}, \max \left\{\left|\frac{\partial^{n} f}{\partial y^{n}}\right|,\left|\frac{\partial^{n} g}{\partial y^{n}}\right|,\left|\frac{\partial^{n} f}{\partial x^{n}}\right|,\left|\frac{\partial^{n} g}{\partial x^{n}}\right|\right\}<M_{2}, \quad \text { and } \\
& \max \left\{\left|\frac{\partial^{m+n} f}{\partial y^{m} \partial t^{n}}\right|,\left|\frac{\partial^{m+n} g}{\partial y^{m} \partial t^{n}}\right|,\left|\frac{\partial^{m+n} f}{\partial x^{m} \partial t^{n}}\right|,\left|\frac{\partial^{m+n} g}{\partial x^{m} \partial t^{n}}\right|\right\}<M_{3}, \forall n, m .
\end{aligned}
$$

Let $M=\max \left\{M_{1}, M_{2}, M_{3}\right\}$,

then, the local truncation errors $T_{i, j}^{(1)}$ of the difference equation (10) will have an upper bound as:

$$
\left|T_{i, j}^{(1)}\right| \leq \frac{M}{2 h k}\left(k\left(a_{1}+a_{2}+b_{1}+b_{2}\right)\left(1+e^{k}\right) \sinh h+(2 h+\varepsilon k)\left(e^{k}-1\right)\right) .
$$

Similarly, the local truncation errors $T_{i, j}^{(2)}$ of the difference equation (11) will have an upper bound as:

$$
\left|T_{i, j}^{(2)}\right| \leq \frac{M}{2 h k}\left(k\left(a_{2}+a_{3}+b_{2}+b_{3}\right)\left(1+e^{k}\right) \sinh h+(2 h+\varepsilon k)\left(e^{k}-1\right)\right) .
$$

Now, in equation (8) if $A$ and $B$ are $3 \times 3$ real symmetric matrices:

$$
A=\left[\begin{array}{lll}
a_{1} & a_{2} & a_{3} \\
a_{2} & a_{3} & a_{4} \\
a_{3} & a_{4} & a_{5}
\end{array}\right] \text {, and } B=\left[\begin{array}{lll}
b_{1} & b_{2} & b_{3} \\
b_{2} & b_{3} & b_{4} \\
b_{3} & b_{4} & b_{5}
\end{array}\right] \text {, }
$$

then the local truncation errors $T_{i, j}^{(1)}, T_{i, j}^{(2)}$ and $T_{i, j}^{(3)}$ for the 3-finite difference equations reduced from equation (8), analogous to equation (10) and (11) can take the form:

$$
\left|T_{i, j}^{(1)}\right| \leq \frac{M}{2 h k}\left(k\left(a_{1}+a_{2}+a_{3}+b_{1}+b_{2}+b_{3}\right)\left(1+e^{k}\right) \sinh h+(2 h+\varepsilon k)\left(e^{k}-1\right)\right),
$$




$$
\begin{aligned}
\left|T_{i, j}^{(2)}\right| & \leq \frac{M}{2 h k}\left(k\left(a_{2}+a_{3}+a_{4}+b_{2}+b_{3}+b_{4}\right)\left(1+e^{k}\right) \sinh h+(2 h+\varepsilon k)\left(e^{k}-1\right)\right), \\
\left|T_{i, j}^{(3)}\right| & \leq \frac{M}{2 h k}\left(k\left(a_{3}+a_{4}+a_{5}+b_{3}+b_{4}+b_{5}\right)\left(1+e^{k}\right) \sinh h+(2 h+\varepsilon k)\left(e^{k}-1\right)\right) .
\end{aligned}
$$

In general if $A$ and $B$ are $n \times n$ real symmetric matrices, the local truncation errors upper bound can take the form:

$$
\begin{aligned}
\left|T_{i, j}^{(m)}\right| \leq \frac{M}{2 h k}( & k\left(a_{m}+a_{m+1}+\cdots+a_{m+n-1}+b_{m}+b_{m+1}+\cdots+b_{m+n-1}\right)\left(1+e^{k}\right) \sinh h \\
& \left.+(2 h+\varepsilon k)\left(e^{k}-1\right)\right), \quad m=1(1) n .
\end{aligned}
$$

\section{Numerical Results}

We present some numerical examples to compare the considered method (10) and (11) with Crank-Nicolson method, and we consider two cases. In case I we apply our method on the examples 1 and 2 such that the exact solution is given at the first level to determine the restrictive parameters $\varepsilon_{11}, \varepsilon_{21}$, and hence we continue using it for another levels for calculation. In the general case the exact solution at the first level is unknown, so in case II we use another method as Crank-Nicolson method, to evaluate the solutions at the first time level by large number of levels by very smali time step length $k$ to determine the restrictive parameters $\varepsilon_{1 i}, \varepsilon_{21}$, after which we can use large time step length $k$ to evaluate the solution at another levels, we apply this case for example 3.

\section{Example1}

$$
\frac{\partial \underline{u}}{\partial t}=A \frac{\partial \underline{u}}{\partial x}+B \frac{\partial \underline{u}}{\partial y}, \quad A=\left[\begin{array}{cr}
-2 & 1 \\
1 & -2
\end{array}\right], B=\left[\begin{array}{cr}
-1 & 0 \\
0 & -1
\end{array}\right],
$$

with $f(x, y, 0), g(x, y, 0), f(0, y, t), g(0, y, t), f(1, y, t), g(1, y, t)$,

and $f(x, 0, t), g(x, 0, t), f(x, 1, t), g(x, 1, t)$, are known from the exact solution:

$$
f(x, y, t)=\sin (x-t)+\sin (y-t), \quad g(x, y, t)=\sin (x-t)+\cos (y-t) .
$$

\section{Example 2}

$$
\frac{\partial \underline{u}}{\partial \boldsymbol{t}}=A \frac{\partial \underline{u}}{\partial x}+B \frac{\partial \underline{u}}{\partial y}, \quad A=\left[\begin{array}{ll}
1 & 0 \\
0 & 1
\end{array}\right], B=\left[\begin{array}{ll}
0 & 1 \\
1 & 0
\end{array}\right],
$$

with $f(x, y, 0), g(x, y, 0), f(0, y, t), g(0, y, t), f(1, y, t), g(1, y, t)$,

and $f(x, 0, t), g(x, 0, t), f(x, 1, t), g(x, 1, t)$, are known from the exact solution: $f(x, y, t)=\cos (x+t)+\cos (y+t), \quad g(x, y, t)=\sin (x+t)+\cos (y+t)$.

\section{Example 3}

$$
\frac{\partial \underline{u}}{\partial t}=A \frac{\partial \underline{u}}{\partial x}+B \frac{\partial \underline{u}}{\partial y}, \quad A=\left[\begin{array}{ll}
1 & 0 \\
0 & 1
\end{array}\right], B=\left[\begin{array}{ll}
0 & 1 \\
1 & 0
\end{array}\right],
$$

with $f(x, y, 0), g(x, y, 0), f(0, y, t), g(0, y, t), f(1, y, t), g(1, y, t)$,

and $f(x, 0, t), g(x, 0, t), f(x, 1, t), g(x, 1, t)$, are known from the exact solution:

$f(x, y, t)=\exp (0.01(x+t))+\exp (0.01(y+t)), g(x, y, t)=\exp (0.01(x+t))+\exp (0.01(y+t))$. 
The numerical results present in this example compare Crank-Nicolson method, with the considered method in equations (10) and (11), in case I and case II, such that in case II we use Crank-Nicolson method to evaluate the solution at the time $t=k=0.9$, by the choice of the time step $k_{1}=0.05$ and $h=0.2$, after 18 step $k=18 \times k_{1}=$ 0.9 , hence we determine the restrictive parameters $\varepsilon_{1 i}, \varepsilon_{2 i}$, then we can use large time step length $k$ to evaluate the solution for another levels, see Table(3).

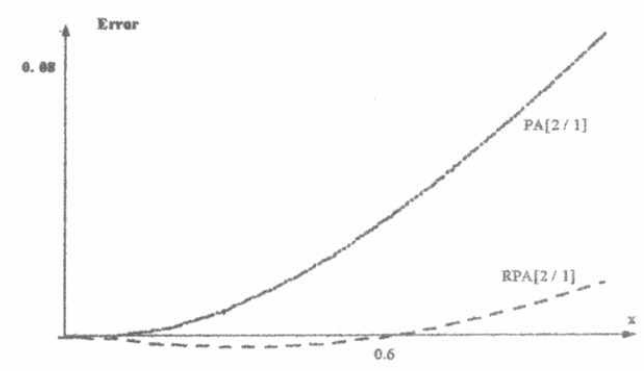

Fig (1)

Comparison of truncation errors between PA[2 / 1] and RPA[2 / 1]

Table(1)

Comparison of the absolute errors (A.E.) and relative errors(R.E.) between Crank-Nicolson and the considered method for $h=0.2$ and $k=.02$, for example 1 .

\begin{tabular}{|l|l|l|l|l|l|}
\hline $\mathrm{t}$ & $(\mathrm{x}, \mathrm{y})$ & \multicolumn{2}{l|}{ Crank-Nicolson method } & \multicolumn{2}{l|}{ The considered method } \\
\hline & & A. E. & R. E. & A. E. & R. E. \\
\hline \multirow{3}{*}{2} & $(0.2,0.2)$ & $2.5 \times 10^{-2}$ & $1.3 \times 10^{-2}$ & $2.6 \times 10^{-15}$ & $1.3 \times 10^{-15}$ \\
& $(0.4,0.4)$ & $5.6 \times 10^{-3}$ & $2.8 \times 10^{-3}$ & $2.6 \times 10^{-15}$ & $1.3 \times 10^{-15}$ \\
& $(0.6,0.6)$ & $1.2 \times 10^{-2}$ & $6.0 \times 10^{-2}$ & $4.4 \times 10^{-16}$ & $2.5 \times 10^{-16}$ \\
& $(0.8,0.8)$ & $2.3 \times 10^{-2}$ & $1.2 \times 10^{-2}$ & $8.4 \times 10^{-15}$ & $4.5 \times 10^{-15}$ \\
\hline \multirow{4}{*}{4} & $(0.2,0.2)$ & $1.9 \times 10^{-2}$ & $1.5 \times 10^{-2}$ & $9.1 \times 10^{-15}$ & $7.4 \times 10^{-15}$ \\
& $(0.4,0.4)$ & $1.2 \times 10^{-3}$ & $1.3 \times 10^{-3}$ & $1.1 \times 10^{-16}$ & $1.2 \times 10^{-16}$ \\
& $(0.6,0.6)$ & $7.7 \times 10^{-3}$ & $1.5 \times 10^{-2}$ & $8.6 \times 10^{-15}$ & $1.6 \times 10^{-14}$ \\
& $(0.8,0.8)$ & $4.6 \times 10^{-4}$ & $4.1 \times 10^{-3}$ & $6.2 \times 10^{-15}$ & $5.3 \times 10^{-14}$ \\
\hline \multirow{6}{*}{8} & $(0.2,0.2)$ & $2.1 \times 10^{-2}$ & $1.0 \times 10^{-2}$ & $2.4 \times 10^{-15}$ & $1.2 \times 10^{-15}$ \\
& $(0.4,0.4)$ & $8.8 \times 10^{-3}$ & $4.5 \times 10^{-3}$ & $2.6 \times 10^{-15}$ & $1.3 \times 10^{-15}$ \\
& $(0.6,0.6)$ & $8.3 \times 10^{-3}$ & $4.6 \times 10^{-3}$ & $2.6 \times 10^{-15}$ & $1.4 \times 10^{-15}$ \\
& $(0.8,0.8)$ & $2.0 \times 10^{-2}$ & $1.2 \times 10^{-2}$ & $1.3 \times 10^{-15}$ & $8.3 \times 10^{-15}$ \\
\hline \multirow{1}{*}{16} & $(0.2,0.2)$ & $2.4 \times 10^{-2}$ & $1.3 \times 10^{-1}$ & $8.7 \times 10^{-15}$ & $4.7 \times 10^{-14}$ \\
& $(0.4,0.4)$ & $6.9 \times 10^{-3}$ & $3.2 \times 10^{-2}$ & $9.0 \times 10^{-15}$ & $4.1 \times 10^{-14}$ \\
& $(0.6,0.6)$ & $7.8 \times 10^{-3}$ & $1.2 \times 10^{-2}$ & $7.2 \times 10^{-15}$ & $1.2 \times 10^{-14}$ \\
& $(0.8,0.8)$ & $7.8 \times 10^{-3}$ & $8.1 \times 10^{-3}$ & $5.8 \times 10^{-15}$ & $6.0 \times 10^{-14}$ \\
\hline
\end{tabular}


Table(2)

Comparison of the absolute errors (A.E.) and relative errors(R.E.) between Crank-Nicolson and the considered method for $h=0.2$ and $k=.02$, for example 2.

\begin{tabular}{|c|c|c|c|c|c|}
\hline$t$ & $(x, y)$ & \multicolumn{2}{c|}{ Crank-Nicolson method } & \multicolumn{2}{c|}{ The considered method } \\
\hline & & A.E. & R. E. & A. E. & R. E. \\
\hline \multirow{4}{*}{2} & $(0.2,0.2)$ & $3.0 \times 10^{-2}$ & $2.5 \times 10^{-2}$ & $3.2 \times 10^{-15}$ & $1.8 \times 10^{-15}$ \\
& $(0.4,0.4)$ & $8.6 \times 10^{-3}$ & $5.8 \times 10^{-3}$ & $5.7 \times 10^{-15}$ & $3.9 \times 10^{-15}$ \\
& $(0.6,0.6)$ & $5.1 \times 10^{-3}$ & $2.9 \times 10^{-3}$ & $5.3 \times 10^{-15}$ & $3.1 \times 10^{-16}$ \\
& $(0.8,0.8)$ & $1.5 \times 10^{-2}$ & $7.9 \times 10^{-3}$ & $3.1 \times 10^{-15}$ & $1.6 \times 10^{-15}$ \\
\hline \multirow{4}{*}{4} & $(0.2,0.2)$ & $1.6 \times 10^{-2}$ & $1.7 \times 10^{-2}$ & $1.5 \times 10^{-14}$ & $1.6 \times 10^{-15}$ \\
& $(0.4,0.4)$ & $1.0 \times 10^{-2}$ & $1.6 \times 10^{-2}$ & $7.7 \times 10^{-16}$ & $1.2 \times 10^{-16}$ \\
& $(0.6,0.6)$ & $8.6 \times 10^{-3}$ & $3.8 \times 10^{-2}$ & $1.0 \times 10^{-14}$ & $4.6 \times 10^{-14}$ \\
& $(0.8,0.8)$ & $2.5 \times 10^{-2}$ & $1.4 \times 10^{-1}$ & $1.3 \times 10^{-15}$ & $7.9 \times 10^{-14}$ \\
\hline \multirow{5}{*}{8} & $(0.2,0.2)$ & $2.3 \times 10^{-2}$ & $3.4 \times 10^{-2}$ & $2.6 \times 10^{-15}$ & $3.9 \times 10^{-15}$ \\
& $(0.4,0.4)$ & $5.5 \times 10^{-3}$ & $5.3 \times 10^{-3}$ & $6.6 \times 10^{-16}$ & $6.4 \times 10^{-15}$ \\
& $(0.6,0.6)$ & $8.2 \times 10^{-3}$ & $6.0 \times 10^{-3}$ & $9.7 \times 10^{-15}$ & $7.1 \times 10^{-15}$ \\
& $(0.8,0.8)$ & $1.0 \times 10^{-2}$ & $6.1 \times 10^{-3}$ & $6.8 \times 10^{-15}$ & $4.2 \times 10^{-15}$ \\
\hline \multirow{1}{*}{16} & $(0.2,0.2)$ & $2.3 \times 10^{-2}$ & $1.3 \times 10^{-2}$ & $1.7 \times 10^{-14}$ & $9.8 \times 10^{-14}$ \\
& $(0.4,0.4)$ & $9.7 \times 10^{-3}$ & $6.3 \times 10^{-3}$ & $2.2 \times 10^{-15}$ & $1.4 \times 10^{-14}$ \\
& $(0.6,0.6)$ & $1.3 \times 10^{-2}$ & $1.0 \times 10^{-2}$ & $1.2 \times 10^{-14}$ & $1.0 \times 10^{-14}$ \\
& $(0.8,0.8)$ & $2.5 \times 10^{-2}$ & $2.8 \times 10^{-2}$ & $2.6 \times 10^{-15}$ & $2.8 \times 10^{-14}$ \\
\hline
\end{tabular}

Table (3)

Comparison of the absolute errors (A.E.) and relative errors(R.E.) between Crank-Nicolson and The considered method for $h=0.2$ and $k=0.9$, for example 3 .

\begin{tabular}{|c|c|c|c|c|c|c|c|}
\hline \multirow[t]{3}{*}{$t$} & \multirow[t]{2}{*}{$x$} & \multirow{2}{*}{\multicolumn{2}{|c|}{$\frac{\text { Crank-Nicolson }}{\text { Method }}$}} & \multicolumn{4}{|c|}{ The considered method } \\
\hline & & & & & & Cas & 11 \\
\hline & & A. E. & R.E. & A.E. & R. E. & A.E. & R.E. \\
\hline 180 & $\begin{array}{l}2,0.2) \\
4,0.4) \\
6,0.6) \\
8,0.8)\end{array}$ & $\begin{array}{l}1.0 \times 10^{-4} \\
4.5 \times 10^{-5} \\
4.5 \times 10^{-5} \\
1.0 \times 10^{-4}\end{array}$ & $\begin{array}{l}9.2 \times 10^{-6} \\
3.9 \times 10^{-6} \\
3.9 \times 10^{-6} \\
9.1 \times 10^{-6}\end{array}$ & $\begin{array}{l}1.9 \times 10^{-13} \\
1.4 \times 10^{-13} \\
1.2 \times 10^{-13} \\
9.7 \times 10^{-14}\end{array}$ & $\begin{array}{l}1.6 \times 10^{-14} \\
1.1 \times 10^{-14} \\
1.0 \times 10^{-14} \\
8.0 \times 10^{-15}\end{array}$ & $\begin{array}{l}9.2 \times 10^{-6} \\
3.9 \times 10^{-6} \\
3.9 \times 10^{-6} \\
9.1 \times 10^{-6}\end{array}$ & $\begin{array}{l}7.6 \times 10^{-7} \\
3.2 \times 10^{-7} \\
3.2 \times 10^{-7} \\
7.5 \times 10^{-7}\end{array}$ \\
\hline 270 & $\begin{array}{l}(0 . \\
(0 . \\
(0 .\end{array}$ & $\begin{array}{l}2.8 \times 10^{-4} \\
1.2 \times 10^{-4} \\
1.2 \times 10^{-4} \\
2.8 \times 10^{-4}\end{array}$ & & $\begin{array}{l}6.6 \times 10^{-13} \\
5.9 \times 10^{-13} \\
4.6 \times 10^{-13} \\
3.5 \times 10^{-13}\end{array}$ & $\begin{array}{l}2.2 \times 10^{-14} \\
1.9 \times 10^{-14} \\
1.5 \times 10^{-14} \\
1.1 \times 10^{-14}\end{array}$ & $\begin{array}{l}2.5 \times 10^{-5} \\
1.0 \times 10^{-5} \\
1.0 \times 10^{-5} \\
2.5 \times 10^{-5} \\
\end{array}$ & $\begin{array}{l}8.5 \times 10^{-7} \\
3.6 \times 10^{-7} \\
3.6 \times 10^{-7} \\
8.4 \times 10^{-7}\end{array}$ \\
\hline 540 & $\begin{array}{l}0 . \\
10 . \\
0 . \\
10 .\end{array}$ & $\begin{array}{l}4.6 \times 10^{-3} \\
1.9 \times 10^{-3} \\
1.9 \times 10^{-3} \\
4.5 \times 10^{-3}\end{array}$ & $\begin{array}{l}1.0 \times 10^{-5} \\
4.4 \times 10^{-6} \\
4.4 \times 10^{-6} \\
1.0 \times 10^{-5}\end{array}$ & $\begin{array}{l}1.3 \times 10^{-12} \\
9.1 \times 10^{-12} \\
6.9 \times 10^{-12} \\
7.2 \times 10^{-12}\end{array}$ & $\begin{array}{l}3.0 \times 10^{-15} \\
2.0 \times 10^{-14} \\
1.5 \times 10^{-14} \\
1.6 \times 10^{-14}\end{array}$ & $\begin{array}{l}4.0 \times 10^{-4} \\
1.7 \times 10^{-4} \\
1.7 \times 10^{-4} \\
4.0 \times 10^{-4}\end{array}$ & $\begin{array}{l}9.1 \times 10^{-7} \\
3.8 \times 10^{-7} \\
3.8 \times 10^{-7} \\
9.0 \times 10^{-7} \\
\end{array}$ \\
\hline 630 & $\begin{array}{l}(0.2,0.2) \\
(0.4,0.4) \\
(0.6,0.6) \\
(0.8,0.8)\end{array}$ & $\begin{array}{l}1.1 \times 10^{-2} \\
4.8 \times 10^{-3} \\
4.8 \times 10^{-3} \\
1.1 \times 10^{-2}\end{array}$ & $\begin{array}{l}1.0 \times 10^{-5} \\
4.4 \times 10^{-6} \\
4.4 \times 10^{-6} \\
1.0 \times 10^{-5}\end{array}$ & $\begin{array}{l}4.4 \times 10^{-11} \\
5.0 \times 10^{-12} \\
5.4 \times 10^{-12} \\
5.5 \times 10^{-11}\end{array}$ & $\begin{array}{l}4.0 \times 10^{-14} \\
4.5 \times 10^{-15} \\
4.9 \times 10^{-15} \\
5.0 \times 10^{-14}\end{array}$ & $\begin{array}{l}9.9 \times 10^{-4} \\
4.2 \times 10^{-4} \\
4.2 \times 10^{-4} \\
9.8 \times 10^{-4}\end{array}$ & $\begin{array}{l}9.1 \times 10^{-7} \\
3.8 \times 10^{-7} \\
3.8 \times 10^{-7} \\
8.0 \times 10^{-7}\end{array}$ \\
\hline
\end{tabular}




\section{Conclusion}

1- The numerical results presented in case I in each of tables (1), (2) and (3) shows that the absolute errors obtained by the considered methods is almost of order $10^{-9}$ of that absolute errors obtained by Crank-Nicolson method.

2- In example 3 we show that the largest absolute error estimation for CrankNicolson method is almost of order $10^{-2}$, while for the considered method using case II the largest absolute error is almost of order $10^{-4}$.

\section{References}

[1] A. R. Mitchell " Computational Methods in Partial Differential Equations" John Wiley \& Sons London New York Sydny Toronto (1969).

[2] Hassan N. A. Ismail and Elsayed M. E. Elbarbary "Restrictive Pade" Approximation and Partial Differential Equation" Int. J. Computer Math. Vol. 66, No. 34 pp. 343-351 (1998).

[3] Hassan N. A. Ismail and Elsayed M. E. M. Elbarbary " Highly Accurate Method for the Convection-Diffusion Equation"Accepted for Publications for Int.J.Computer Math.Vol. 74, No 3.

[4] Hassan N.A.Ismail, Elsayed M.E.M.Elbarbary and Adel Younes Hassan " Highly Accurate Method for Solving Initial Boundary Value Problem for First Order Hyperbolic Differential Equations " to Appear in Int.J.Computer Math.

[5] Hassan N.A.Ismail, and Adel Younes Hassan "Restrictive Pade' Approximation for Solving First Order Hyperbolic Partial Differential Equations " Accepted for Publication in J. of Institute of Math. \& Computer Sciences Vol. 11 No. 1 (2000).

[6] Hassan N.A.Ismail, Elsayed M.E.M.Elbarbary and Adel Younes Hassan "Restrictive Pade' Approximation for Solving First Order Hyperbolic Systems in One Space Dimension " to Appear in Int.J.Computer Math.

[7] Hassan N.A.lsmail, and Adel Younes Hassan "Restrictive Pade" Approximation for Solving First Order Hyperbolic Partial Differential Equations in Two space Dimensions " to Appear in J. of Institute of Math. \& Computer Sciences. 